\title{
UV transmittance in market place sunglasses and their adherence to established standards
}

\author{
Daisuke Serizawa $^{1}$, Helen Heacock ${ }^{2}$ \\ 1 Lead Author, B. Tech Student, School of Health Sciences, British Columbia Institute of Technology, 3700 \\ Willingdon Ave, Burnaby, BC, V5G 3H2 \\ 2 Supervisor, School of Health Sciences, British Columbia Institute of Technology, 3700 Willingdon Ave, Burnaby, \\ BC, V5G 3H2
}

\begin{abstract}
Background: Sunglasses are used to shade and protect the public's eyes every day. However some improperly made sunglasses offer inadequate UV protection that shade the eyes and dilate pupils, while letting in a high dose of UV radiation into sensitive ocular tissues. This UV exposure can have acute and chronic effects such as temporary blindness and clouding of the eye. This study investigated the prevalence of sunglasses with poor UV protection and examined any relationships or associations between such sunglasses and their retail price or declared protective standards. Methods: 35 unused sunglasses available in the Metro Vancouver area were tested using an Agilent 8453 UV-visible Spectroscopy System for UV transmittance rates in the UVA, UVB, and UVC wavelengths. Results were statistically analyzed for any potential relationships or associations between price, price categories, total number of wavelengths failed, transmittance test results, decal presence, and types of decals present. Results: Sample sunglasses were distributed to be $51 \%$ budget sunglasses, $23 \%$ standard sunglasses, and $26 \%$ premium sunglasses. Of these 35 sunglasses, $11 \%$ failed the $4 \%$ permitted transmittance test, and $89 \%$ of the sunglasses had some form of UV protection claim adhered or printed on the product. Statistically significant associations, using Chi-squared analysis, could not be found between transmittance test results and price category, UV protection claims, or the type of UV protection claim; p-values were found to be $0.43643,0.44525$, and 0.58402 , respectively. A statistically significant relationship, using linear regression, could not be found between price and total wavelengths failed; $p$-value was found to be 0.2272 with a slope of -0.1334 Conclusion: Though no statistically significant relationships or associations could not be found, the study did find sunglasses that offered inadequate UV protection, leading to the conclusion that there are sunglasses in the Metro Vancouver market that are inappropriate for standard UV protection.
\end{abstract}

Keywords: UV protection, sunglasses, sunglass, UVR, UV, photoprotection, Vancouver, ocular health, vision

\section{Introduction}

Hazardous effects of ultraviolet (UV) radiation exposure have been studied thoroughly in the past half century. Such research has focused on UV exposure from various sources on various organs, with chronic and acute exposure being taken into account as well. With mounting evidence specifically 
tying UVB exposure as well as UVA exposure to the formation of cataracts, photokeratitis, and cystoids macular edema, investigation into UV exposure effects on ocular tissue is extremely important. It is equally important to recognize that protective eyewear such as sunglasses aid in the reduction of UV exposure to ocular tissue. In this proposal, such protective qualities of sunglasses that consumers depend upon were investigated to determine the quality of ophthalmic protection currently on the market. Sunglasses from both the "low-budget" and "designer" categories were examined to determine their UVA and UVB absorption rates, and compared to their respective declared protection. The high volume of counterfeit and budget sunglasses in the market today are ambiguous in providing protection for the sensitive organs that we rely on every day. The hope is that continued research into the prevalence of sub-quality sunglasses will lead to an enforceable national or international standard.

\section{Literature Review}

\section{UV Radiation}

UV radiation is categorized into three different types via their respective wavelengths. UVC refers to the shortest wavelength group, with the most energy, thus most potential to inflict harm to tissues. Its wavelength is between $100 \mathrm{~nm}$ to $280 \mathrm{~nm}$. UVB constitutes radiation in the $280 \mathrm{~nm}$ to $320 \mathrm{~nm}$ range. UVA spans $320 \mathrm{~nm}$ to $400 \mathrm{~nm}$ and is the closest to visible light in terms of wavelength.

The sun is the greatest source of environmental exposure to UV radiation, emitting all three forms of UV radiation (Health Canada, n.d.). However, the earth's ozone layer absorbs all UVC, allowing only UVB and UVA radiation to reach the earth's surface. The makeup of this UV is approximately $96.5 \%$ UVA and 3.5\% UVB (Almutawa, Vandal, Wang, \& Lim, 2013). The exposure hazard of this bombardment of radiation increases around reflective surfaces such as snow, metal, and water, all of which can reflect nearly $85 \%$ of incident radiation (Rubin \& Fishman, 1986). This poses a particular hazard for alpine sports enthusiasts and recreational outdoor water users who expose themselves to increased UV radiation compared to the rest of the population.
Susceptible populations will be visited later in this literature review.

\section{Health Effects of UV Exposure}

The dangers of UV exposure have and continue to be well documented in the medical community. UV exposure has been tied to melanoma, cataracts, photokeratitis, a drop in keratopathy, as well as macular degeneration (Almutawa et al., 2013). Photokeratitis is a condition of the eye in response to acute overexposure to UV, while the other conditions previously mentioned are due to chronic exposure. UV radiation has shorter wavelengths than visible light, and therefore has more energy than visible light. This increases potential for damage that UV can inflict relative to visible light (Health Canada, n.d.). Sunglasses offer protection via reflection of visible light and absorption of UV radiation, and conversion of absorbed energy into heat (Abney \& Scalettar, 1998). In order to offer adequate protection, such sunglasses need to absorb both UVA as well as UVB.

\section{UV Exposure and Pathology}

UVB and UVA have been demonstrated to have different mechanisms of inflicting damage to ocular tissue. Both recent as well as older studies have tied both UVB and UVA with reduced photoreceptor sensitivity in the retina, formation of pterygium and pinguelcula, macular edema, and macular degeneration (Laube, Apel, \& Koch, 2004). An epidemiological review of evidence has shown that UV irradiated individuals were 1.4 times more likely to develop cataracts (McCarty \& Taylor, 2002). Skin damage has been seen to arise at different times after UV exposure. UVA radiation affects the skin with a sudden but temporary sunburn that alleviates after two to three hours, while UVB is responsible for the "day-after" sunburn that manifests between 12 and 36 hours (Abney \& Scalettar, 1998). UVB irradiation results in the conversion of individual UV absorption molecules, within the eye, into free radicals that cause oxidative damage to the lens and cornea (Rubin $\&$ Fishman, 1986). The toxic nature of free radicals can be attributed to their natural structure; unpaired electrons in their outer orbital rim make them unstable and readily reactive. The photochemical damage induced by UV radiation occurs much more 
easily than thermal damage, delaying an individual's recognition that damage has been done (Rubin \& Fishman, 1986). Chronic UVB radiation exposure has been implicated in formation of cataracts, a condition characterized by the opacification of the lens, while acute exposure causes photokeratitis as mentioned earlier (Kullavanijaya \& Lim, 2005). Blue light is also a spectrum of radiation that is under scrutiny by the scientific community. Though it is not studied as widely, some suggest that routine exposure can age parts of the eye leading to macular degeneration and increase the risk of blindness and photosensitivity (Health Canada, n.d.) (Almutawa et al., 2013). Blue light is visible light in the $400 \mathrm{~nm}$ to $440 \mathrm{~nm}$ wavelength that is responsible for inhibiting lens focus. It is very prevalent in reflections from surfaces such as snow and water (Health Canada, n.d.).

\section{Anatomic Defenses and Susceptible Populations}

Immune and regeneration capabilities of ocular tissue post-UV radiation are variable due to different structures absorbing respective wavelengths. Surface layers (cornea and conjunctiva) absorb UVB, the lens absorbs UVA, and visible light is absorbed by the retina. To combat toxic free radicals that form after irradiation, the absorptive tissues utilize antioxidants to prevent damage. Antioxidants divert potential damage from free radicals by reacting with them to form neutral products. However, under heavy irradiation, thus heavy oxidative stress, normal antioxidant levels may be inadequate in protecting tissues (Kullavanijaya \& Lim, 2005). The problem is compounded with age since antioxidant levels have been observed to decrease with age. Furthermore, the study by Kullavanaijaya \& Lim (2005) noted that “...major ocular tissues such as lens and retina do not possess the capacity of cellular regeneration; therefore, damaged molecules accumulate during one's lifetime." This reinforces findings that individuals who have had cataract extraction or ocular surgery and are above the age of 50 are at increased risk of ocular tissue damage from UV radiation (Diaz \& Nesbitt, 2013). Diaz \& Nesbitt (2013) also identified individuals who spend time near surface waters, snow, or at high altitudes as individuals with higher than normal exposure to UV radiation. This was attributed to reflected incident radiation from surfaces having an additive effect on solar radiation, doubling the normal exposure levels. Airline pilots are also among higher risk populations due to their exposure to both cosmic ionizing and UV radiation (Rafnsson et al., 2005). For the general population solar UV radiation is seen as a hazard throughout the day for skin but for the eyes exposure is highest at 08:00-10:00 and 14:00-16:00 (American National Standards Institute, n.d.). The American National Standards Institute recommends the use of sunglasses in the previously mentioned times due to solar radiation being in the same plane as the general population's eyes. Seasonally, most people expect to use UV protection in the summer months but Environment Canada recommends the use of protective sunglasses in the spring and fall as well. Environment Canada's UV Index guideline recommends wearing sunglasses on bright days with a UV index of 0-2, though the index can go as high as 11(Environment Canada, 2013).

\section{Public Knowledge of UV Radiation}

A telephone survey conducted in 1994 indicated that the general public's knowledge of the effects of sunlight on the eyes was mediocre (Lee, Hirst, \& Sheehan, 1994). The study indicates that the public had reasonable knowledge of health effects of sunlight on the eyes, with a $92 \%$ correct response rate, but when questioned about specific diseases and their relation to UV exposure, the correct response rate fell to less than a third. The study was conducted with 500 individuals in Australia, and similar studies have followed suit, all indicating fair to good knowledge of the need for eye protection when exposed to sunlight (Wright, Reeder, Gray, \& Cox, 2008)(Ahn, Frederikson, Borman, \& Bednarek, 2011). However, studies conducted outside of Australia and New Zealand could not be identified. Observational studies on the use of sunglasses outdoors have been conducted in Australia and Hawaii, with a study population of 15,261 and 5,171 respectively (Lagerlund et al., 2006) (Maddock, O'Riordan, Lee, Mayer, \& McKenzie, 2009). These studies indicate that in some high UV exposure countries there is a lack of knowledge on the relationship of UV exposure and eye health, as well as a lack in the use of sunglasses as sufficient protection. The use of sunglasses and eye protection 
should be integrated into public health promotion and the standards for sunglasses should be developed and enforced.

\section{Market Sunglasses and Mechanism for Ocular Damage}

Sunglasses on the market today come in various colors, tints, shapes, sizes, thickness, and prices. However, none of these factors are sufficient indicators for the level of UV protection they offer; consumers cannot visually assess their protective properties (Health Canada, n.d.). Lenses with darker tint have the ability to decrease the amount of visible light that can reach the eyes. The decreased light reaching the eyes causes the pupils to dilate, which essentially increases the opening of the eyes for more radiation, both UV and visible, to enter the eye. Without adequate UV protection in the lenses of sunglasses, higher levels of UV radiation will enter the eyes than otherwise not wearing such sunglasses at all (American National Standards Institute, n.d.). Some older studies have investigated the transmission rates of UV radiation in market sunglasses and found UV transmission rates from $1.5 \%$ to nearly $40 \%$ (Rosenthal, Bakalian, Lou, \& Taylor, 1988). Some studies revealed $47 \%$ of sunglasses were not satisfactory for UV protection (Leow \& Tham, 1995).

\section{Consumer Protection Standards}

There are several standards currently employed around the world to standardize quality and protection of sunglasses in the world today; three national standards and one international standard. ANSI Z80.3, EN 1836, and AS/NZS1067 are the American, European, and Australian standards, while the international standard is ISO 12312-1. Though these standards offer similar requirements of protection, only the European standard and the Australian standards are legally mandated to be followed, of which only the Australian standard is the only one enforced by third party testing (Almutawa et al., 2013). Health Canada, responsible for product safety for sunglasses sold in Canada, currently does not conduct third party testing of sunglasses. They require manufacturers to follow industry standards and recommends consumers to look for "UV400" or "CE" labeled products which indicate compliance to ANSI Z80.3 and EN 1836 standards, respectively
(Health Canada, n.d.). Currently there is an absence of federal and provincial legislation mandating labeling of sunglasses or testing of sunglasses. As Health Canada recommends, looking for "UV400" or "CE" declaration on products is the only way for consumers to find proper eye protection. The ANSI Z80.3 standard will be used to assess sunglasses for this study due to its specific UV spectrum transmittance limits.

\section{Public Health Significance}

Protection of the public's vision is an important aspect of public health. Sunglasses must possess UV absorption capabilities that are considered safe such as those outlined in the above mentioned national and international standards. Should Canada adopt legislation that enforces such standards or develop their own standards, the inspection of such products would be conducted by Health Canada and federal inspectors mandated by legislation. Such testing of consumer sunglasses is currently conducted by the Optics and Radiometry Laboratory (ORLAB) for the Australian market and would be a worthwhile source of information for testing products at the federal level (Stephen John Dain et al., 2010). In a 2010 study, ORLAB tested 646 pairs of sunglasses with "CE" designation. Of the sunglasses tested $17.3 \%$ failed, with $1.8 \%$ of sunglasses having excessive UV transmittance (Stephen John Dain et al., 2010). As mentioned previously, UV protection cannot be determined by price, color, or darkness of tint. Enforcement of standards through third party testing should be adopted to protect the vision of Canadian consumers. The investigation of sunglasses and their respective standards to protect ocular health possesses public health significance and should be further investigated.

\section{Study Purpose}

The lack of UV protection in sunglasses can cause both short term and long term health risks when used in the presence of UV radiation. The purpose of this study was to investigate the adherence of declared UV transmission rates to their actual UV transmission rates, and their potential relationship to price. The results of this study can be indicative of the performance of sunglasses on the market today, 
and if there is a need for individuals to look for standard conformity declarations or seals.

\section{Methods and Materials}

\section{Introduction and Current Standards}

UV transmittance through sunglasses has been studied in various research articles and standards for such transmittance have been developed in a handful of countries. These standards include three national standards, as well as an international standard that has been developed for recommended transmittance values. The international standard, ISO 12312-1, and American standard, ANSI Z80.3 both are offered as a standard for producers as well as consumers (American National Standards Institute, 2008; International Organization for Standardization, 2013). In comparison, the Australian and European standards, AS/NZS 1067 and EN 1836, are both legal requirements for sunglasses sold in their respective regions, though the Australian standard is the only one to be enforced with third party testing. Analysis methods of transmittance currently employed in established standards and academic research can be grouped into two general categories: radiometric and spectrophotometric. Radiometric analysis assesses transmittance as watts per surface area, with no relation to the strength of the source light. Spectrophotometric analysis is the standardized method used for the previously mentioned standards. All standards have required transmittance levels prescribed as luminous transmittance or a fraction thereof.

ANSI Z80.3 states that UV must be no greater than half of the luminous transmittance allowance of $8 \%$ to $40 \%$. The strictest permissible luminous transmittance of $8 \%$ would mean that UV transmittance must be below $4 \%$ to be of passing grade. For calculation purposes, a stricter transmittance of $4 \%$ was used to test sample sunglasses for their ability to absorb/transmit UV. Due to research constraints, other recognized standards were not be used for transmittance testing.

The right lens of the sample sunglasses were tested in the spectrophotometer; two separate readings were taken after which the average of the two was recorded per each tested wavelength. All sample sunglasses were tested for the wavelengths $200 \mathrm{~nm}$ to $400 \mathrm{~nm}$ and any wavelength that failed the $4 \%$ maximum transmittance rate were recorded as a failed wavelength, and any sunglasses with a failed wavelength was recorded as a failed sample. The ALIGENT 8453 Spectrophotometer was used to test all sample sunglasses that fit the inclusion/exclusion criteria below.

\section{Inclusion and Exclusion Criteria}

\begin{tabular}{|c|}
\hline Inclusion Criteria \\
\hline 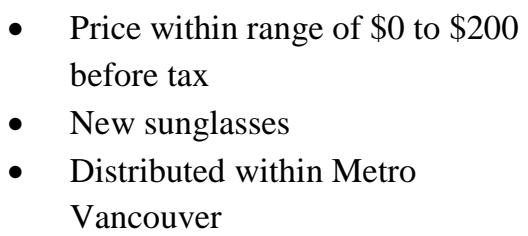 \\
\hline Exclusion Criteria \\
\hline $\begin{array}{ll}\text { - } & \text { Used sunglasses } \\
\text { - } & \text { Soiled lenses }\end{array}$ \\
\hline
\end{tabular}

\section{Results}

\section{Organization}

The following types of values were collected; price, wavelengths failed, and (protection) decal present. These values were further processed as follows:

- Price was stratified into 3 distinct price categories

○ Price Category 1: $\$ 0.00$ to $\$ 9.99$

Budget Sunglasses

○ Price Category 2: $\$ 10.00$ to $\$ 99.99$

Standard Sunglasses

○ Price Category 3: $\$ 100.00$ to $\$ 200.00$

Premium Sunglasses

- Wavelengths failed were compiled to form test results

○ PASS: No wavelength falls below the $4 \%$ transmittance allowance

0 FAIL: Any wavelength falls below the $4 \%$ transmittance allowance 
- Decal present was further dissected to three different decal types

- NONE: No UV protection claim present

- UV400: UV400 claim present, claiming compliance with ANSI Z80.3

- CE: CE claim present, claiming compliance with EN 1836

o BOTH: Both CE and UV400 claims present

Statistical analysis was conducted using Microsoft Excel for data entry purposes and descriptive analysis while NCSS 9 was used for inferential analysis (Hintze, 2013; Microsoft Corporation, 2010).

\section{Descriptive Statistics}

The price of the 35 samples of sunglasses had a mean of $\$ 46.68$ while the median was $\$ 5.00$. Given that the mode was found to be $\$ 4.00$, it can be concluded that sunglasses had mostly lower prices while some higher priced sunglasses shifted the mean farther/higher from the median. The lower value of the median compared to the mean is also an indication that the sunglass prices were skewed to the left, which is further reinforced by the mode value. Spread is relatively wide since standard deviation of $\$ 60.35$ is rather close to the mean of $\$ 46.68$, and the range was found to be $\$ 190$ between a minimum of $\$ 0.00$ and a maximum of $\$ 190.00$.

Table 1 Collected Data and Test Results

\begin{tabular}{|lll|}
\hline $\begin{array}{l}\text { Price Category and } \\
\text { Range }\end{array}$ & Count & Percent of Total \\
1) $\$ 0.00$ to $\$ 9.99$ & 18 & $51 \%$ \\
2) $\$ 10.00$ to $\$ 99.99$ & 8 & $23 \%$ \\
3) $\$ 100.00$ to $\$ 200.00$ & 9 & $26 \%$ \\
\hline Test Result & Count & Percent of Total \\
FAIL & 4 & $11 \%$ \\
PASS & 31 & $89 \%$ \\
\hline Decal Presence & Count & Percent of Total \\
No Decal Present & 4 & $11 \%$ \\
Decal Present & 31 & $89 \%$ \\
\hline Type of Decal & Count & Percent of Total \\
UV400 & 9 & $26 \%$ \\
CE & 15 & $43 \%$ \\
NONE & 4 & $11 \%$ \\
BOTH & 7 & $20 \%$ \\
\hline
\end{tabular}

The price categories were separated into 3 categories as seen in the previous table. In this study of 35 sunglasses, the largest portion of the sunglasses belonged to the lowest price category; category 1 . Table 1 Collected Data and Test Results show that of the 35 sunglasses tested, only $11 \%$ failed to comply with the permitted $4 \%$ transmittance.

Decal presence had identical distribution as the test results with an 89\%:11\% ratio as represented in Table 1 Collected Data and Test Results. It is important to note however that though the ratios were identical, the relationship between test results and the presence of a decal was not identical. This will be further investigated in the discussion. The table indicates the proportions of sunglasses that had UV protection claims "CE", "UV400", no claims, or both "CE" and "UV400” claims.

\section{Inferential Statistics}

Further analysis of data was conducted using NCSS 9, specifically to run linear regression and correlation, as well as Chi-square tests. Justifications for choices of tests are given below.

\section{Linear Regression and Correlation}

$\mathrm{H}_{\mathrm{o}}$ : Slope $=0$; There is no relationship between price and total wavelengths failed.

$\mathrm{H}_{\mathrm{A}}$ : Slope $\neq 0$; There is a relationship between price and total wavelengths failed.

Both numeric values of total wavelengths failed and price was analyzed using linear regression and correlation. Linear correlation was ideal to analyze whether there was a relationship between total wavelengths failed and price since both values were numerical. Investigation of price as the independent variable to total wavelengths failed as the dependent variable revealed that, though there are outlying points, there is a negative correlation between price and total wavelengths failed. Linear regression and correlation analysis indicated that the linear relationship can be represented as the following: 
Total Wavelengths Failed $=(-0.1334)$ Price +18.1131

Formula 2 Linear Regression

This result is consistent with the previously mentioned suggestion of a negative correlation between price and total wavelengths failed, as indicated by the slope of -0.1334 in Formula 2 Linear Regression. Using this formula, one can predict the total wavelengths a sunglass will fail in a transmittance test in the sampled population by inputting Price and solving for Total Wavelengths Failed in Formula 2 Linear Transmission. The formula also allows for the prediction of a minimum price for an acceptable pair of sunglasses. To have sunglasses that do not fail any wavelengths for the $4 \%$ transmittance allowance, the minimum price is predicted to be approximately $\$ 135.78$. However this relationship was found to not possess strong correlation or significance to accurately predict a minimum price. Statistical analysis went on to show that the $r$ value, correlation coefficient, has a value of -0.2095 ; an indicator of a low degree of relationship between the two variables. A significance test returned a value of 0.2272 as the significance level. Since 0.2272 is greater than $0.05, \mathrm{H}_{0}$ cannot be rejected and it cannot be concluded that the slope is not zero.

\section{Chi-Squared Test}

\section{Price Category VS Test Results}

Analysis of price category and test results, both nonnumeric values, was conducted using Chi-square test. Test results were deemed as the dependent variable while price category was the independent variable, since price category was expected to influence test results of samples.

$\mathrm{H}_{\mathrm{o}}$ : There is no association between price category and whether or not sunglasses pass the transmission test.

$\mathrm{H}_{\mathrm{A}}$ : There is an association between price category and whether or not sunglasses pass the transmission test.

Pearson's Chi-Square test produced a p-value of 0.43643 ; a value greater than the threshold of 0.05 .
The p-value does not allow for the rejection of $\mathrm{H}_{\mathrm{o}}$ and therefore it cannot be concluded that there is an association between price category and whether or not sunglasses pass the transmission test.

\section{Decal Present VS Test Results}

Analysis of decal presence and test results, both nonnumeric values, was conducted using Chi-square test. Test results were deemed as the dependent variable while decal presence was the independent variable, since decal presence was expected to influence test results of samples.

$\mathrm{H}_{\mathrm{o}}$ : There is no association between decal presence and whether or not sunglasses pass the transmission test.

$\mathrm{H}_{\mathrm{A}}$ : There is an association between decal presence and whether or not sunglasses pass the transmission test.

Pearson's Chi-Square test produced a p-value of 0.44525 ; a value greater than the threshold of 0.05 . The $\mathrm{p}$-value does not allow for the rejection of $\mathrm{H}_{\mathrm{o}}$ and therefore it cannot be concluded that there is an association between decal presence and whether or not sunglasses pass the transmission test.

\section{Type of Decal VS Test Results}

Analysis of type of decal and test results, both nonnumeric values, was conducted using Chi-square test. Test results were deemed as the dependent variable while type of decal was the independent variable, since the type of decal was expected to influence test results of samples.

$\mathrm{H}_{\mathrm{o}}$ : There is no association between type of decal and whether or not sunglasses pass the transmission test.

$\mathrm{H}_{\mathrm{A}}$ : There is an association between type of decal and whether or not sunglasses pass the transmission test.

Pearson's Chi-Square test produced a p-value of 0.58402 ; a value greater than the threshold of 0.05 . The $\mathrm{p}$-value does not allow for the rejection of $\mathrm{H}_{\mathrm{o}}$ and therefore it cannot be concluded that there is an 
association between type of decal and whether or not sunglasses pass the transmission test.

\section{Potential Errors and Power of the Study}

The p-value found in the linear regression, 0.2272, is lower than threshold value of 0.05 . Due to the high $\mathrm{p}$ value, a determination of the alpha error is not possible since an alpha error requires the rejection of the null hypothesis. This also applies to the Chisquare tests which produced p-values of 0.43643 , 0.44525 , and 0.58402. According to the linear regression analysis, the power of the study was 0.2229 , a value less than the desired threshold of 0.80 to minimize beta error. This is indicative of significant potential for beta error and it can be attributed to the low sample size. This beta error could be limited by increasing the number of sample sunglasses to have a power above 0.80 .

\section{Discussion}

Though statistical analysis returned results that were not statistically significant, it is profound that of 35 sunglasses available to consumers in the Metro Vancouver area 4 sunglasses failed. This is an overall fail rate of $11.4 \%$ of the sampled sunglasses. Though the samples were from several brands and several different retail establishments, it is discerning to find that $11 \%$ of sunglasses obtained in Metro Vancouver fail the $4 \%$ permitted transmittance limit. UV protection of individual sunglasses can only be communicated to the public through the presence of protection claim decals. $11 \%$ of sunglasses obtained for this study had no UV protection decals present, and therefore leave the public unable to determine if their sunglasses protect them at all. $11 \%$ of tested sunglasses failed the transmittance tests, and by coincidence $11 \%$ of tested sunglasses did not have decals present. However these were not the same sunglasses; all of the sunglasses that did not have a decal passed the transmittance tests. It can be assumed from these results that the presence or absence of decals is not indicative of UV protection. This is supported in the Chi-square analysis of test results and decal presence, which produced a p-value of 0.44525 , and failed to reject the null hypothesis.
The investigation of decal type and transmittance test results failed to establish a statistically significant association between the two variables. The high pvalue of 0.58402 indicated the transmittance test results are independent of the type of decal present on sunglasses. This, similar to the result seen between transmittance test results and decal presence, defeats the purpose of protection decals ensuring adequate protection. The highest proportion of failed sunglasses had the "UV400" decal, with 2 out of 7 tested sunglasses failing the transmittance test.

The role of price did not have a statistically significant relationship or association with UV protection as well. Though Chi-square test results seemed to show an association between price category and test results, with 3 out of 4 test failures belonging to the lowest price category, the analysis returned a p-value of 0.43643 . Therefore the hypothesis that price category and test results are independent could not be rejected. Similar results were found when linear regression was used to investigate the relationship between price and total wavelengths failed. A p-value of 0.2272 was returned for the slope of the relationship, which fails to reject the null hypothesis that there is a statistically significant relationship between price and total wavelengths failed. Though the linear equation produced from the analysis can be used to determine the hypothetical price for UV protective sunglasses, further investigation is required, desirably with an increased sample size to lower beta error, to produce a model with statistical significance.

From the above analysis of the results, it is not possible to say with certainty that price or UV protection claims are adequate indicators of protection. Similar studies have taken place in the past, returning results that indicated various proportions of market sunglasses that had inadequate protection. An Australian study that tested 646 sunglasses found that $1.8 \%$ had excessive UV transmittance, a lower percentage than the $11 \%$ fail rate of this study(Stephen John Dain et al., 2010). Some older studies have returned varied results such as UV transmittance rates of $1.5 \%$ to as high as $40 \%$ (Rubin \& Fishman, 1986), or an almost perfect compliance rate from a test of 30 sunglasses (Blumthaler \& Ambach, 1991), while an examination 
of children's and toy sunglasses in 1985 returned a UV protection failure rate of $36 \%$ and $33 \%$ respectively(S J Dain, Hearne, \& Pepper, 1985). Leow \& Tham (1995) also investigated 34 sunglasses and when compared with their respective prices, it was found that price was not indicative of UV protection. A more recent study from 2007 involving 16 sunglasses specifically used in dermal phototherapy and found that most performed at adequate levels(Dongre, Pai, \& Khopkar, 2007). This study further went on to investigate how branded and non-branded sunglasses performed. It was concluded that branding was not a proficient indicator of UV protection from the results, as there were passing and failing sunglasses from both branded and nonbranded groups.

Relevant past studies as well as the results from this study indicate two situations; there currently are unacceptable sunglasses on the market place today and that certain common qualities such as price are not indicative of proper protection. The results of this study further reinforce the common hypothesis and evidence that such common qualities are unreliable and at times misleading for the general public in choosing proficient eye wear. It is imperative that there be an indicator that the public can rely to ensure smart choices about protection can be made. It is the shared responsibility of public health professionals to safeguard the public's ocular health. It is the stance of Health Canada that the sunglasses sold in Canada are aligned with the standards and certifications of ANSI, and that the producers are legally mandated to comply with such standards. However, as mentioned previously, the enforcement of these legally mandated standards are not conducted. The stance of Health Canada, the results of previous research, and the results of this current study, call for a new directive or policy that will ensure that mandated minimums are met. It would be ideal to have a third party similar to that of Australia's ORLAB to test sunglasses, as this would not only help protect the general public, but also the many outdoor enthusiasts who are involved in activities that can have much higher UV exposures. Though this study could not prove relationships between UV protection and other variables, it is clear that unacceptable sunglasses are on the market, and actions should be taken to combat the production and distribution of such products.

\section{Limitations}

There were several clear limitations of this study. Financial restrictions made the acquisition of sample sunglasses difficult. A total of 35 sunglasses were able to be tested but stronger results, potentially with statistically significant results, could be assumed to have been observed had there been more samples available for testing. This would have also helped increase the power of the study and in-turn decrease beta error. Another limitation of this study was that the UV protection claim decals are stickers and can be added or transferred between sunglasses. This allows for distributors and retailers and even consumers to change the declared protection rates of the sunglasses. Most CE declarations were permanently printed on the inside arms of sunglasses to combat this problem, but many of the declarations were in the form of removable/transferable stickers on the lenses of the sunglasses. Lastly, due to unavailable data on sunglass sales in Metro Vancouver, the determination of the proper representative sample size was not possible.

\section{Conclusion}

This study finds that there was no statistically significant relationship between price and UV protection of sunglasses available in Metro Vancouver. Results do not allow for the accurate prediction of a safe price of sunglasses to gain adequate UV protection. It was also found that there was no statistically significant association between UV protection and price category, UV protection claim decal presence, or type of decal present. However it can be concluded that there are non-fully UV protective sunglasses in the Metro Vancouver market from the presence of 4 sunglasses that failed UV transmittance tests.

\section{Recommendations and Future Studies}

As mentioned previously, the presence of inadequate sunglasses is clear from the results of this study. In order to keep such products off of Metro Vancouver and Canadian markets, legislation should be enacted to mandate adherence to accepted protection standards such as ANSI Z80.3 and CE 1836. As the 
"Public Health Significance" section of this paper points out, if such legislation and enforcement of protection standards materialize, it would become the responsibility of Health Canada and public health professionals. It is the main recommendation of this study that further research be conducted to better understand the prevalence of inadequate sunglasses in the Metro Vancouver and Canadian market. An investigation of the same parameters but with a larger sample size as well as a larger variety of retailers and brands would be ideal. Not only would this be a more accurate representation of the market, but it would also add power to the statistical analysis. It can be said that Dr. Stephen Dain has lead the field, most notably with a similar study of 646 sunglasses in Australia. A study of such magnitude conducted in other countries would be able to shed light on this rather under-investigated topic. Such research would further contribute to the field of public health and ocular protection. As well, this would help lobby for the adoption of the more stringent recommendations and policies in the future.

\section{Acknowledgements}

I would like to thank Kevin Soulsbury for graciously providing the facilities and spectrophotometric expertise to conduct my tests, $\mathrm{Vu}$ Nguyen for providing support, and Helen Heacock for guidance, advice, and expertise throughout the span of this project. I would also like to extend my gratitude to Barrette Roach, Liam McCarthy, Debbie Lee, and Howard Chen for providing sample sunglasses for this study.

\section{Competing Interest}

The authors declare that there are no competing interests.

\section{References}

Abney, J. R., \& Scalettar, B. a. (1998). Saving Your Students' Skin. Undergraduate Experiments that Probe UV Protection by Sunscreens and
Sunglasses. Journal of Chemical Education, 75(6), 757. doi:10.1021/ed075p757

Ahn, M. J., Frederikson, L., Borman, B., \& Bednarek, R. (2011). Eye health in New Zealand: A study of public knowledge, attitudes, and practices related to eye health and disease. Health Education, 111(2), 147-155. doi:10.1108/09654281111108562

Almutawa, F., Vandal, R., Wang, S. Q., \& Lim, H. W. (2013). Current status of photoprotection by window glass, automobile glass, window films, and sunglasses. Photodermatology, Photoimmunology \& Photomedicine, 29(2), 65-72. doi:10.1111/phpp.12022

American National Standards Institute. (n.d.). Media Tips and Case Studies Sunglasses. Retrieved October 03, 2013, from http://www.ansi.org/news_publications/media_t ips/sunglasses.aspx

American National Standards Institute. (2008). Nonprescription Sunglasses and Fashion Eyewear Requirements: ANSI Z80.3. New York: American National Standards Institute.

Blumthaler, M., \& Ambach, W. (1991). How well do sunglasses protect against ultraviolet radiation? Lancet, 337(8752), 1284. Retrieved from http://www.ncbi.nlm.nih.gov/pubmed/1674077

Dain, S J, Hearne, G., \& Pepper, L. (1985). The Performance of Toy Sunglasses and Children's Sunglasses. Australian Journal of Optometry, 68(5), 183-186.

Dain, Stephen John, Ngo, T. P. T., Cheng, B. B., Hu, A., Teh, A. G. B., Tseng, J., \& Vu, N. (2010). Sunglasses, the European directive and the European standard. Ophthalmic \& Physiological Optics: The journal of the British College of Ophthalmic Opticians (Optometrists), 30(3), 253-6. doi:10.1111/j.1475-1313.2010.00711.x 
Diaz, J. H., \& Nesbitt, L. T. (2013). Sun exposure behavior and protection: recommendations for travelers. Journal of Travel Medicine, 20(2), 108-18. doi:10.1111/j.1708-8305.2012.00667.x

Dongre, A. M., Pai, G. G., \& Khopkar, U. S. (2007). Ultraviolet protective properties of branded and unbranded sunglasses available in the Indian market in UV phototherapy chambers. Indian $J$ Dermatol Venereol Leprol, 73(1), 26-28.

Environment Canada. (2013). Sun Protection Messages. Retrieved October 03, 2013, from http://www.ec.gc.ca/uv/default.asp?lang=En\&n $=\mathrm{DCF} 1 \mathrm{C} 20 \mathrm{~A}-1$

Health Canada. (n.d.). It's Your Health. Retrieved October 04, 2013, from http://www.hcsc.gc.ca/hl-vs/iyh-vsv/prod/glasses-lunetteseng.php

Hintze, J. (2013). NCSS 9 - Student License. Kaysville, Utah: NCSS, LLC. Retrieved from www.ncss.com

International Organization for Standardization. (2013). ISO 12312-1:2013 Eye and face protection - Sunglasses and related eyewear Part 1: Sunglasses for general use. Geneva: International Organization for Standardization.

Kullavanijaya, P., \& Lim, H. W. (2005). Photoprotection. Journal of the American Academy of Dermatology, 52(6), 937-58; quiz 959-62. doi:10.1016/j.jaad.2004.07.063

Lagerlund, M., Dixon, H. G., Simpson, J. a, Spittal, M., Taylor, H. R., \& Dobbinson, S. J. (2006). Observed use of sunglasses in public outdoor settings around Melbourne, Australia: 1993 to 2002. Preventive medicine, 42(4), 291-6. doi:10.1016/j.ypmed.2006.01.003

Laube, T., Apel, H., \& Koch, H.-R. (2004). Ultraviolet radiation absorption of intraocular lenses. Ophthalmology, 111(5), 880-5. doi:10.1016/j.ophtha.2003.08.031
Lee, G., Hirst, L., \& Sheehan, M. (1994). Knowledge of sunlight effects on the eyes and protective behaviors in the general community. Ophthalmic Epidemiology, 1, 67-84.

Leow, Y. H., \& Tham, S. N. (1995). UV-protective sunglasses for UVA irradiation protection. International Journal of Dermatology, 34(11), 808-10. Retrieved from http://www.ncbi.nlm.nih.gov/pubmed/8543419

Maddock, J. E., O’Riordan, D. L., Lee, T., Mayer, J. a, \& McKenzie, T. L. (2009). Use of sunglasses in public outdoor recreation settings in Honolulu, Hawaii. Optometry and Vision Science: Official Publication of the American Academy of Optometry, 86(2), 165-6. doi:10.1097/OPX.0b013e318194eae7

McCarty, C. A., \& Taylor, H. R. (2002). A review of the Epidemiologic evidence linking ultraviolet radiation and cataracts. Developmental Opthalmology, 35, 21-31.

Microsoft Corporation. (2010). Microsoft Excel (Version 2010). Microsoft Corporation.

Rafnsson, V., Olafsdottir, E., Hrafnkelsson, J., Sasaki, H., Arnarsson, A., \& Jonasson, F. (2005). Cosmic Radiation Increases the Risk of Nuclear Cataract in Airline Pilots, 123.

Rosenthal, F. S., Bakalian, a E., Lou, C. Q., \& Taylor, H. R. (1988). The effect of sunglasses on ocular exposure to ultraviolet radiation. American Journal of Public Health, 78(1), 72$4 . \quad$ Retrieved from http://www.pubmedcentral.nih.gov/articlerende r.fcgi?artid=1349214\&tool=pmcentrez\&rendert ype $=$ abstract

Rubin, M. L., \& Fishman, G. A. (1986). PERSPECTIVES Ocular Phototoxicity :, 31(2).

Wright, C., Reeder, A. I., Gray, A., \& Cox, B. (2008). Child sun protection: sun-related attitudes mediate the association between children's knowledge and behaviours. Journal 
of Paediatrics and Child Health, 44(12), 692

8. doi:10.1111/j.1440-1754.2008.01408.x 